\title{
Relationships between Dietary Inflammatory Index score and markers of inflammation and WNT signalling in the healthy colorectal mucosa
}

\author{
F.C. Malcomson ${ }^{1}$, N.D. Willis ${ }^{1}$, I. McCallum ${ }^{2}$, N. Shivappa ${ }^{3}$, M. Wirth ${ }^{3}$, J. Hébert ${ }^{3}$, \\ B. Kocaadam ${ }^{4}$, A. Özturan ${ }^{5}$, S. Kelly ${ }^{2}$, D.M. Bradburn ${ }^{6}$, N.J. Belshaw ${ }^{7}$, I.T. Johnson ${ }^{8}$ and \\ J.C. Mathers ${ }^{1}$ \\ ${ }^{1}$ Human Nutrition Research Centre, Institute of Cellular Medicine, Newcastle University, Framlington Place, \\ Newcastle upon Tyne, NE2 4HH, UK, \\ ${ }^{2}$ Northumbria Healthcare NHS Foundation Trust, North Shields, NE29 8NH, UK, \\ ${ }^{3}$ Arnold School of Public Health, University of South Carolina, Columbia, South Carolina, USA, \\ ${ }^{4}$ Gazi University, Faculty of Health Sciences, Department of Nutrition and Dietetics, Ankara, Turkey, \\ ${ }^{5}$ Adnan Menderes University, Faculty of Health Sciences, Department of Nutrition and Dietetics, Aydin, Turkey, \\ ${ }^{6}$ Northumbria Healthcare NHS Foundation Trust, Ashington, NE63 9JJ, UK, \\ ${ }^{7}$ University of East Anglia, Norwich Research Park, Norwich, NR4 7TJ, UK and \\ ${ }^{8}$ Quadram Institute, Norwich Research Park, Norwich, NR4 7UQ, UK
}

Chronic inflammation is associated with increasing the risk of colorectal cancer (CRC), and both inflammation and CRC risk are modulated by environmental factors such as diet. The Dietary Inflammatory Index $\left(\mathrm{DII}{ }^{\circledR}\right.$ ) provides a tool to assess the inflammatory potential of diet and DII score has been linked with several health outcomes ${ }^{(1)}$. For example, individuals with a high DII score (more inflammatory diet) have been shown to have a $40 \%$ increased risk of CRC compared with those with the lowest DII scores ${ }^{(2)}$.

The WNT signalling pathway is involved in the maintenance of homeostasis within the large bowel through the regulation of physiological processes, such as cell proliferation, but is also implicated in colorectal carcinogenesis. Furthermore, interactions between WNT signalling and inflammatory pathways exist ${ }^{(3)}$.

The aim of this study was to investigate relationships between the inflammatory effects of diet, assessed using the DII, and markers of inflammation and of the WNT signalling pathway in the healthy colorectal mucosa.

We used samples and dietary data from 75 healthy participants recruited to the DISC Study ${ }^{(3)}$. Energy-adjusted (E-DII) scores were calculated using food frequency questionnaire data and included 30 food parameters. Systemic inflammation was assessed by quantifying high-sensitivity C-reactive protein (hsCRP) and local inflammation from faecal calprotectin. Expression and methylation of WNT pathway genes were quantified in rectal mucosal biopsies by qPCR and by pyrosequencing, respectively. Correlation and regression analyses between E-DII score and the measured outcomes were performed. Participants were divided into pro- and antiinflammatory groups according to E-DII score by dichotimising at the median $(0.700)$ for categorical analyses using the ANOVA General Linear Model and adjusting for age, gender, BMI, smoking status and endoscopy procedure as covariates. Corresponding non-parametric tests were applied where required.

Mean E-DII score within DISC Study participants was 0.736 (range -4.480-5.030). hsCRP concentrations were significantly greater in the pro-inflammatory E-DII group (median $=3.0)$ compared with the anti-inflammatory group $($ median $=1.6)(p=0.03)$. Positive relationships between E-DII and expression of FOSL1 $(\mathrm{p}=0.006)$ and $W N T 11(\mathrm{p}=0.008)$ were observed. Rectal WNTI1 expression was significantly higher in the pro-inflammatory group (Least Squares Mean $(\mathrm{LSM})=0.344$ ) compared with the antiinflammatory group $(\mathrm{LSM}=0.243)(\mathrm{p}=0.003)$.

The findings from this study provide evidence for relationships between pro-inflammatory diets (greater DII score) and markers of inflammation and WNT signalling in the healthy colorectal mucosa. Aberrant expression of FOSL1 and WNT11, which increased with greater E-DII scores, has been reported in CRC and associated with effects on functional outcomes such as cell proliferation and migration which are dysregulated in CRC. These findings suggest that WNT signalling may be a mechanism through which diet, particularly inflammatory components of diet, modulate large bowel health and CRC risk.

We acknowledge the staff at the gastroenterology units at Wansbeck and North Tyneside General Hospitals for their help and support with participant recruitment and study visits. We also thank the DISC Study participants without whom this study would have been impossible.

1. Shivappa N, Steck SE, Hurley TG et al. (2014) Public Health Nutr 17, 1689.

2. Shivappa N, Godos J, Hebert J et al. (2017) Nutrients 9(9), 1043.

3. Moparthi L and Koch S (2019) Differentiation 108, 24.

4. Malcomson FC, Willis ND, McCallum I et al. (2017) AJCN 105, 400. 